\title{
Streamlined Synthesis of 6-((1H-1,2,3-Triazol-4-yl)methyl)-1H-pyrrolo [3,4-d]pyridazin-1-one System via Sequential $N$-Alkylation, CuAAC, and [4 + 2] Cyclization Reactions
}

\author{
Helio G. Bonacorso, ${ }^{\circledR *, a}$ Hernane T. Magalhães, ${ }^{a}$ Gean M. Dal Forno, ${ }^{a}$ \\ Francieli M. Libero, ${ }^{a}$ Manfredo Hoerner, ${ }^{a}$ Clarissa P. Frizzo, ${ }^{a}$ Marcos A. P. Martins ${ }^{a}$ \\ and Nilo Zanatta ${ }^{a}$
}

${ }^{a}$ Núcleo de Química de Heterociclos (NUQUIMHE), Departamento de Química, Universidade Federal de Santa Maria, 97105-900 Santa Maria-RS, Brazil

\begin{abstract}
An efficient sequential three-step reaction methodology for the synthesis of three new series1-(prop-2-yn-1-yl)-1H-pyrroles, methyl 4-acetyl-1-((1H-1,2,3-triazol-4-yl)methyl)-1H-pyrrole3-carboxylates and 6-((1H-1,2,3-triazol-4-yl)methyl)-2,6-dihydro- $1 H$-pyrrolo[3,4-d]pyridazin1-ones-is reported. The methodology comprises: (i) $\mathrm{N}$-alkylation reactions of polyfunctionalized $1 H$-pyrroles-which were previously obtained from $(E)$-methyl 2-azido-3-arylacrylates-with propargyl bromide in order to obtain $1 \mathrm{H}$-pyrroles; (ii) standard copper-catalyzed azide-alkyne cycloaddition (CuAAC) involving organic azides (benzyl-, 4-methoxybenzyl- and 4-chlorobenzyl, as well as $n$-octyl azide) and $\mathrm{N}$-propargylated $1 H$-pyrroles to give triazolyl derivatives, as methyl $1 H$-pyrrole-3-carboxylates (click chemistry); and (iii) [4+2] cyclocondensation reactions of the ketoesters in the presence of hydrazine hydrochloride in order to furnish the desired series of pyrrolo[3,4- $d]$ pyridazin-1-ones at total yields up to $54 \%$.
\end{abstract}

Keywords: pyrroles, triazoles, pyrrolo[3,4-d]pyridazinones, $N$-alkylation, CuAAC, cyclocondensation reaction

\section{Introduction}

Heterocyclic compounds play a significant role in synthetic chemistry. In particular, the pyrrolo[3,4- $d]$ pyridazinone system has been extensively explored in the literature, due to its anticancer activity and being a strong analgesic agent and used in the treatment of autoimmune and immunologically mediated diseases. ${ }^{1-6}$ Thus, the addition, fusion, or derivatization of pyrrolopyridazinones to or from other heterocycles is an important way of obtaining new molecules with high potential for biological activity. ${ }^{7-11}$

Polysubstituted pyrroles ${ }^{12-14}$ are widely used in the treatment of several important diseases. For example, atorvastatin is a drug used to prevent cardiovascular diseases and it has become one of world's best-selling drugs. ${ }^{13,14}$

In this context, due to the high metabolic stability, propensity to make hydrogen bond, dipole-dipole,

*e-mail: helio.bonacorso@ufsm.br and $\pi$ stacking interactions with biological targets, 1,2,3-triazole nucleus has become one of the most promising pharmacological scaffolds for the development of new drugs. In the last few decades, triazole derivatives have been used in the development of new pharmaceutical agents possessing anti-cancer, anti-fungal, anti-malarial, anti-diabetic, anti-leishmanial, anti-tripanossomal and acetylcholinesterase inhibitory properties (Figure 1). ${ }^{15-23}$ 1,2,3-Triazoles are generally obtained through a copper-catalyzed azide-alkyne cycloaddition (CuAAC) reaction, which is an easy and widely used synthetic methodology. ${ }^{24-27}$ Figure 1 shows examples of synthetic heterocycles containing polysubstituted pyrrole, triazole and pyrrolo[3,4- $d]$ pyridazinone rings with pharmacological activity.

Despite all the studies, documents and patents mentioning the numerous biological properties of pyrrolo[3,4- $d$ ] pyridazinone derivatives and 1,2,3-triazoles, no methodology has been developed in recent decades for the combination of these heterocycles as diheteroaryl methylene-spacer systems. Thus, due to our background in the synthesis of pyrroles ${ }^{28-32}$ and 
<smiles>CC(C)c1c(C(=O)Nc2ccccc2)c(-c2ccccc2)c(-c2ccc(F)cc2)n1CC[C@H](O)C[C@@H](O)CC(=O)O</smiles>

Atorvastatin (Cholesterol-lowering)<smiles>C=CC=CCc1ccc(C(=O)c2ccc(C)cc2)n1C</smiles>

Tolmetin (Anti-inflammatory)<smiles>CNCc1cc(-c2ccccc2F)n(S(=O)(=O)c2cccnc2)c1</smiles>

Vonoprazan

(Antacid)<smiles>[R]OCCn1cc(C(N)=O)nn1</smiles><smiles>NC1C(=O)N2C(C(=O)O)=C(CSc3c[nH]nn3)CSC12</smiles>
(Antibiotic)<smiles>NC(=O)C(N)c1ccc(O)cc1</smiles><smiles>CC(F)(F)OC(=O)OC(O)(Cn1cncn1)Cn1cncn1</smiles><smiles>Cc1c2c(O)nn(CCN3CCN(c4ccccc4F)CC3)c(=O)c2c(C)n1-c1ccccc1</smiles><smiles>CC(C)Cc1nn(C)c(=O)c2cn(Cc3cccc4ccccc34)cc12</smiles>

Figure 1. Known synthetic heterocycles containing polysubstituted pyrrole, triazole, and pyrrolo[ $3,4-d]$ pyridazinone nuclei with pharmacological activity.

pyrrolo $[3,4-d]$ pyridazinones,${ }^{33}$ we envisioned the possibility of using an efficient methodology for the synthesis of a new series of a more complex heterocyclic system, which could have a wide range of biological activities for further applications.

In this work the concise sequential three-step reaction methodology for the synthesis of new series of 6-((1H-1,2,3-triazol-4-yl)methyl)-2,6-dihydro$1 H$-pyrrolo[3,4- $d$ ] pyridazin-1-ones (12-15) is disclosed. The method starts from an $\mathrm{N}$-alkylation reaction of polyfunctionalized pyrroles (2)-previously obtained from $(E)$-methyl 2-azido-3-arylacrylates $(\mathbf{1})$-with propargyl bromide, in order to obtain 1-(prop-2-yn-1-yl)$1 H$-pyrroles (3). Subsequently, a standard CuAAC reaction involving organic azides (generated in situ) and pyrroles 3 gives the methyl 4-acetyl-1-(( $1 H-1,2,3$-triazol4-yl)methyl)- $1 H$-pyrrole-3-carboxylates 8-11, and, finally, a $[4+2]$ cyclocondensation reaction of the triazolyl methylene-spacer pyrroles 8-11 with hydrazine hydrochloride furnishes the desired pyrrolo $[3,4-d]$ pyridazin1-one system 12-15 at total yields of up to $54 \%$. Scheme 1 shows an overview of the synthetic strategy for obtaining all the novel heterocycles proposed in this work.

\section{Experimental}

Unless otherwise indicated all common reagents and solvents were used as obtained from commercial suppliers without further purification. ${ }^{1} \mathrm{H}$ and ${ }^{13} \mathrm{C}$ nuclear magnetic resonance (NMR) spectra were acquired on a Bruker DPX 200 spectrometer $\left({ }^{1} \mathrm{H}\right.$ at $200.13 \mathrm{MHz}$ and ${ }^{13} \mathrm{C}$ at $\left.50.32 \mathrm{MHz}\right)$ or Bruker DPX 400 spectrometer $\left({ }^{1} \mathrm{H}\right.$ at $400.13 \mathrm{MHz}$ and ${ }^{13} \mathrm{C}$ at $\left.100.61 \mathrm{MHz}\right), 5 \mathrm{~mm}$ sample tubes, $298 \mathrm{~K}$, digital resolution $\pm 0.01 \mathrm{ppm}$, in $\mathrm{CDCl}_{3}$ using tetramethylsilane (TMS) as internal reference. All melting points were determined using coverslips on an Microquímica MQAPF-302 apparatus and are uncorrected. For the ${ }^{13} \mathrm{C}$ NMR experiments, the chemical shifts were calibrated using a residual non-deuterated solvent as an internal reference. All results are reported as follows: chemical shift $(\delta)$ (multiplicity, integration, coupling constant). The following abbreviations were used to explain multiplicities: $\mathrm{s}=$ singlet, $\mathrm{d}=$ doublet, $\mathrm{t}=$ triplet, $\mathrm{q}=$ quartet, qui $=$ quintet, $\mathrm{m}=$ multiplet, $\mathrm{dd}=$ doublet of doublets. All NMR chemical shifts are reported in parts per million relative to the internal reference. Gas chromatography-mass spectrometry (GC-MS) analyses 


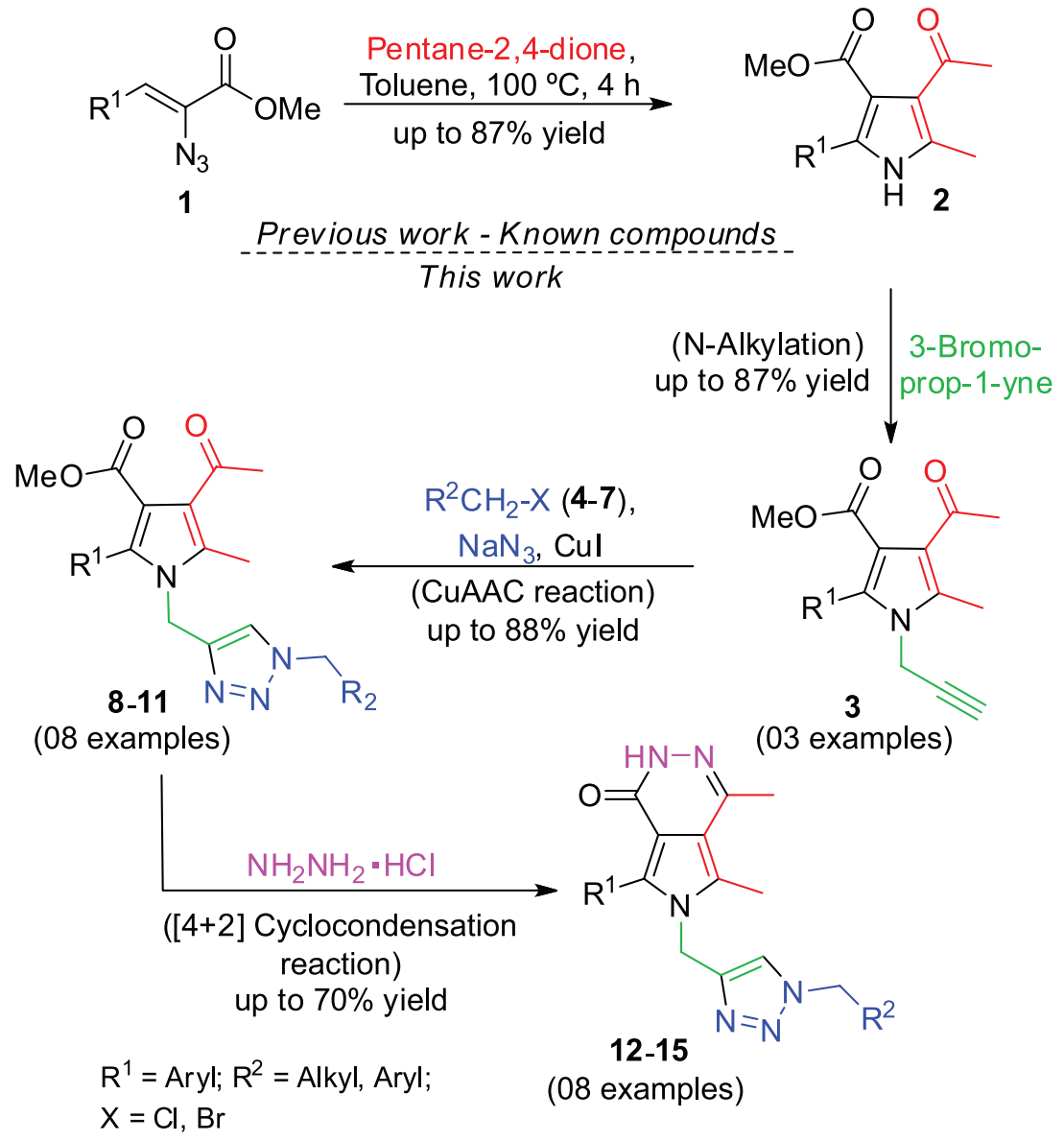

Scheme 1. An overview of the synthetic strategy for the proposed work.

were registered with a split-splitless injector, autosampler, capillary column ( $30 \mathrm{~m}, 0.32 \mathrm{~mm}$ internal diameter), and helium was used as the carrier gas. The CHN elemental analyses were performed on a PerkinElmer $2400 \mathrm{CHN}$ elemental analyzer (Universidade de Santa Cruz do Sul, Brazil).

High-resolution mass spectra (HRMS) were obtained for all compounds on an LTQ Orbitrap Discovery mass spectrometer (Thermo Fisher Scientific). This hybrid system combines an LTQ XL linear ion-trap mass spectrometer and an Orbitrap mass analyzer. The experiments were performed via direct infusion of the sample (flow rate $10 \mu \mathrm{L} \mathrm{min}^{-1}$ ) in positive-ion mode using electrospray ionization (ESI). Elemental composition calculations were executed using the specific tool included in the Qual Browser module of the Xcalibur (Thermo Fisher Scientific, release 2.0.7) software.

$\mathrm{X}$-ray diffraction data for a white single crystal of 1-(prop-2-yn-1-yl)-1 $H$-pyrrole (3a) and for a white single crystal of 1-((1H-1,2,3-triazol-4-yl)methyl)-1H-pyrrole (8a) were performed on a Bruker D8 QUEST and on a Bruker D8 VENTURE, equipped with a PHOTON 100 CMOS detector and, a $\mathrm{Cu}_{\mathrm{K} \alpha}$ I $\mu$ s micro-focus source $(\lambda=1.54178 \AA)$ and a $\mathrm{Ag}_{\mathrm{K} \alpha}$ I $\mu$ s micro-focus source $(\lambda=0.56086 \AA)$, respectively. Indexing was performed using APEX3 software package. ${ }^{34}$ Data integration and reduction were executed using SAINTPLUS 6.01 program. ${ }^{35}$ Absorption correction was performed by multi-scan method implemented in SADABS program. ${ }^{36}$ Respective space groups of the crystal systems of $\mathbf{3 a}$ and 8a were determined using XPREP program integrated in APEX $3 .{ }^{34}$ The structure of both compounds were solved by direct methods contained in Sir2014 v. $17.01^{37}$ and refined on $\mathrm{F}^{2}$ with anisotropic temperature parameters for all non $\mathrm{H}$ atoms using SHELXL version 2016/6 $6^{38}$ integrated in WinGX version 2014.1 system. ${ }^{39}$ Hydrogen atoms were located in geometrically calculated positions (aromatic group: $\mathrm{C}-\mathrm{H}=0.93 \AA$ for $\mathrm{C}_{\mathrm{sp}}{ }^{2}$ atoms; methyl groups: $\mathrm{C}-\mathrm{H}=0.96 \AA$ for $\mathrm{C}_{\mathrm{sp}}{ }^{3}$ atoms; propargyl group: $\mathrm{C}-\mathrm{H}=0.93 \AA$ for $\mathrm{C}_{\mathrm{sp}}$ atom) and treated as riding on their respective $\mathrm{C}$ atoms, with $U_{\text {iso }}(\mathrm{H})$ values set at $1.2 U_{\mathrm{eq}} \mathrm{C}_{\mathrm{sp}}{ }^{2}$ (aromatic and propargyl fragments) and $1.5 U_{\mathrm{eq}} \mathrm{C}_{\mathrm{sp}}{ }^{3}$ (methyl substituents). The crystallographic parameters and details of data collection and refinement are listed on Tables S1 and S4 (Supplementary Information (SI) section). Selected bond and angles are listed on Tables S2 and S5 (SI section). 
Graphical representations involved the DIAMOND program version $3.1 \mathrm{a}^{40}$

General procedure for the synthesis of 1-(prop-2-yn-1-yl)$1 \mathrm{H}$-pyrroles (3a-b)

In a round-bottomed flask, the respective pyrrole (2a-b) $(1 \mathrm{mmol})$ and $\mathrm{K}_{2} \mathrm{CO}_{3}(4 \mathrm{mmol}, 0.55 \mathrm{~g})$ were solubilized in dimethylformamide (DMF, $5 \mathrm{~mL}$ ) at room temperature. Pure propargyl bromide $(1.2 \mathrm{mmol}, 0.14 \mathrm{~g})$ was then added slowly under ice bath at $0-5{ }^{\circ} \mathrm{C}$. The mixture was magnetically stirred at $65^{\circ} \mathrm{C}$ for $5 \mathrm{~h}$. After this time, $40 \mathrm{~mL}$ of water was added at room temperature to the reaction mixture, and this mixture was then washed with ethylacetate $(3 \times 20 \mathrm{~mL})$. The combined organic fractions were washed with water $(3 \times 20 \mathrm{~mL})$ and then with $\mathrm{NaCl}$ aqueous saturated solution $(20 \mathrm{~mL})$. The organic solution was dried with anhydrous $\mathrm{Na}_{2} \mathrm{SO}_{4}$ and then filtered, and the solvent was then evaporated under reduced pressure. The crude solid products $\mathbf{3 a}$-b were obtained at good purity grade at $85-87 \%$ yields, and were used for the next reaction without previous purification.

Methyl 4-acetyl-5-methyl-2-phenyl-1-(prop-2-yn-1-yl)$1 \mathrm{H}$-pyrrole-3-carboxylate (3a)

White solid; yield 85\%; mp 120-122 ${ }^{\circ} \mathrm{C}$; ${ }^{1} \mathrm{H}$ NMR $\left(400 \mathrm{MHz}, \mathrm{CDCl}_{3}\right) \delta$ 7.42-7.26 (m, 5H), 4.35 (d, 2H), 3.58 (s, 3H), 2.48 (s, 3H), 2.41 (s, 3H), 2.37 (t, 1H); ${ }^{13} \mathrm{C}$ NMR $\left(100 \mathrm{MHz}, \mathrm{CDCl}_{3}\right) \delta 198.0,165.4,136.8,133.2,130.6$, 130.3, 129.0, 128.3, 123.4, 113.2, 77.6, 73.7, 51.4, 34.3, 31.0, 11,2; GC-MS (EI, $70 \mathrm{eV}) \mathrm{m} / \mathrm{z}(\%) 295\left(\mathrm{M}^{+}, 30\right), 280$ (32), 262 (100), 220 (25), 206 (21), 192 (20), 181 (9); HRMS (ESI-TOF) $m / z$, calcd. for $\mathrm{C}_{18} \mathrm{H}_{18} \mathrm{NO}_{3}[\mathrm{M}+\mathrm{H}]^{+}$: 296.1287; found: 296.1277.

Methyl 4-acetyl-5-methyl-2-(4-methoxyphenyl)-1-(prop-2-yn1-yl)-1 $H$-pyrrole-3-carboxylate (3b)

Orange solid; yield 87\%; mp 119-122 ${ }^{\circ} \mathrm{C}$; ${ }^{1} \mathrm{H}$ NMR $\left(400 \mathrm{MHz}, \mathrm{CDCl}_{3}\right) \delta$ 7.36-7.34 (m, 2H), 6.99-6.97 (m, $2 \mathrm{H}), 4.38(\mathrm{~d}, 2 \mathrm{H}), 3.86(\mathrm{~s}, 3 \mathrm{H}), 3.63(\mathrm{~s}, 3 \mathrm{H}), 2.50(\mathrm{~s}, 3 \mathrm{H})$, 2.43 (s, 3H), $2.40(\mathrm{t}, 1 \mathrm{H}) ;{ }^{13} \mathrm{C}$ NMR $\left(100 \mathrm{MHz}, \mathrm{CDCl}_{3}\right)$ $\delta$ 198.1, 165.5, 160.0, 136.8, 132.9, 132.0, 123.3, 122.2, 113.7, 112.9, 77.7, 73.6, 55.3, 51.4, 34.2, 31.0, 11.2; HRMS (ESI-TOF) $m / z$, calcd. for $\mathrm{C}_{19} \mathrm{H}_{20} \mathrm{NO}_{4}[\mathrm{M}+\mathrm{H}]^{+}: 326.1392$; found: 326.1379 .

General procedure for the synthesis of methyl 4-acetyl1-((1H-1,2,3-triazol-4-yl)methyl)-1H-pyrrole-3-carboxylates (8-11)

To a stirred solution of $\mathrm{H}_{2} \mathrm{O} / \mathrm{t}-\mathrm{BuOH} 1: 1 \mathrm{v} / \mathrm{v}(12 \mathrm{~mL})$ at room temperature, the respective pyrrole $(\mathbf{3 a}-\mathbf{b}, 1 \mathrm{mmol})$, alkyl halide (4-7, $1.2 \mathrm{mmol})$, and $\mathrm{NaN}_{3}(1.2 \mathrm{mmol}$, $0.078 \mathrm{~g}$ ) were added as one portion. After $30 \mathrm{~min}$ under magnetic stirring, $\mathrm{CuI}(15 \mathrm{mmol} \%)$ was also added at room temperature. The reaction mixture was then heated at $80{ }^{\circ} \mathrm{C}$ for $4 \mathrm{~h}$. Water $(40 \mathrm{~mL})$ was then added to the reaction mixture at room temperature and this mixture was then washed with ethylacetate $(3 \times 20 \mathrm{~mL})$. The combined organic fractions were washed with water $(3 \times 20 \mathrm{~mL})$ and then with $\mathrm{NaCl}$ aqueous saturated solution $(20 \mathrm{~mL})$. The organic solution was dried with anhydrous $\mathrm{Na}_{2} \mathrm{SO}_{4}$ and then filtered, and the solution was then concentrated under reduced pressure. The products 8-11 were purified by column chromatography on silica gel, using a solution of ethyl acetate (30\%) in hexane as eluent. Complete spectral data for compounds 8-11 are available at SI section.

Methyl 4-acetyl-1-((1-benzyl-1H-1,2,3-triazol-4-yl)methyl)5-methyl-2-phenyl-1 $\mathrm{H}$-pyrrole-3-carboxylate (8a)

White solid; yield $77 \%$; mp $130-134{ }^{\circ} \mathrm{C}$; ${ }^{1} \mathrm{H}$ NMR $\left(400 \mathrm{MHz}, \mathrm{CDCl}_{3}\right) \delta$ 7.39-7.34 (m, 4H), 7.31-7.26 (m, 2H), 7.20-7.16 (m, 4H), 6.77 (s, 1H), $5.43(\mathrm{~s}, 2 \mathrm{H}), 4.98(\mathrm{~s}, 2 \mathrm{H})$, 3.57 (s, 3H), 2.43 (s, 3H), 2.41 (s, 3H); ${ }^{13} \mathrm{C} \mathrm{NMR}(100 \mathrm{MHz}$, $\left.\mathrm{CDCl}_{3}\right) \delta 198.5,165.4,144.2,143.4,137.0,133.3,130.8$, $130.7,129.3,129.0,128.3,128.1,123.5,121.8,113.4$, 54.3, 51.5, 40.1, 31.2, 11.6; GC-MS (EI, $70 \mathrm{eV}) \mathrm{m} / \mathrm{z}$ (\%) 429 ( $\left.\mathrm{M}^{+}, 18\right), 355$ (32), 281 (46), 221 (23), 147 (29), 133 (10); calcd. for $\mathrm{C}_{25} \mathrm{H}_{24} \mathrm{~N}_{4} \mathrm{O}_{3}$ : C 70.08, H 5.65, N 13.08\%, found: C 70.35, H 5.69, N 13.10\%.

Methyl 4-acetyl-1-((1-benzyl-1H-1,2,3-triazol-4-yl)methyl)5-methyl-2-(4-methoxyphenyl)-1 $H$-pyrrole-3-carboxylate (8b)

Yellow solid; yield $86 \%$; mp 124-129 ${ }^{\circ} \mathrm{C}$; ${ }^{1} \mathrm{H}$ NMR (400 MHz, $\left.\mathrm{CDCl}_{3}\right) \delta 7.38$ (s, 4H), 7.20 (s, 2H), 7.09-7.08 (m, 2H), 6.87 (s, 1H), 6.81-6.80 (m, 1H), 5.44 (s, 2H), 4.99 (s, 2H), $3.80(\mathrm{~s}, 3 \mathrm{H}), 3.58(\mathrm{~s}, 3 \mathrm{H}), 2.41(\mathrm{~s}, 3 \mathrm{H}), 2.39$ (s, $3 \mathrm{H}) ;{ }^{13} \mathrm{C} \mathrm{NMR}\left(100 \mathrm{MHz}, \mathrm{CDCl}_{3}\right) \delta 198.5,165.5,160.1$, 137.0, 134.4, 133.1, 132.0, 129.8, 129.3, 129.1, 128.1, 123.4, 122.7, 121.9, 113.8, 113.4, 55.4, 54.4, 51.4, 39.9, 31.1, 11.6; HRMS (ESI-TOF) $m / z$, calcd. for $\mathrm{C}_{26} \mathrm{H}_{27} \mathrm{~N}_{4} \mathrm{O}_{4}$ $[\mathrm{M}+\mathrm{H}]^{+}:$459.2032; found: 459.2032 .

Methyl 4-acetyl-1-((1-(4-methoxybenzyl)-1H-1,2,3-triazol4-yl)methyl)-5-methyl-2-phenyl-1 $H$-pyrrole-3-carboxylate (9a)

White solid; yield $60 \%$; mp $135-136{ }^{\circ} \mathrm{C} ;{ }^{1} \mathrm{H}$ NMR $\left(400 \mathrm{MHz}, \mathrm{CDCl}_{3}\right) \delta$ 7.37-7.28 (m, 3H), 7.16-7.10 (m, 4H), $6.87(\mathrm{~d}, 2 \mathrm{H}), 6.79$ (s, 1H), 5.32 (s, 2H), 4.94 (s, 2H), 3.77 (s, 3H), 3.53 (s, 3H), 2.39 (s, 3H), 2.38 (s, 3H); ${ }^{13} \mathrm{C}$ NMR $\left(100 \mathrm{MHz}, \mathrm{CDCl}_{3}\right) \delta 198.7,165.6,160.0,159.9,146.9$, $137.7,133.6,132.2,132.1,129.5,126.1,123.3,122.8$, 
114.3, 113.8, 113.3, 55.4, 53.9, 51.4, 40.5, 31.2, 11.8; HRMS (ESI-TOF) $m / z$, calcd. for $\mathrm{C}_{26} \mathrm{H}_{27} \mathrm{~N}_{4} \mathrm{O}_{4}[\mathrm{M}+\mathrm{H}]^{+}$: 459.2032; found: 459.2040 .

Methyl 4-acetyl-1-((1-(4-methoxybenzyl)-1H-1,2,3-triazol4-yl)methyl)-5-methyl-2-(4-methoxyphenyl)-1 H-pyrrole3-carboxylate (9b)

Yellow solid; yield 70\%; mp 120-122 ${ }^{\circ} \mathrm{C}$; ${ }^{1} \mathrm{H}$ NMR (400 MHz, $\mathrm{CDCl}_{3}$ ) $\delta$ 7.23-7.18 (m, 4H), 6.93 (s, 1H), 6.90 (d, 2H), 6.81 (d, 2H), 5.49 (s, 2H), 4.90 (s, 2H), 3.82 (s, $3 \mathrm{H}), 3.80$ (s, 3H), 3.60 (s, 3H), 2.45 (s, 3H), 2.40 (s, 3H); ${ }^{13} \mathrm{C} \mathrm{NMR}\left(100 \mathrm{MHz}, \mathrm{CDCl}_{3}\right) \delta 198.7,165.6,159.9,159.8$, 137.7, 133.6, 132.2, 132.1, 129.5, 126.1, 123.3, 122.8, 114.3, 113.9, 113.8, 113.3, 55.3, 55.1, 53.9, 51.4, 40.5, 31.2, 11.8; HRMS (ESI-TOF) $m / z$, calcd. for $\mathrm{C}_{27} \mathrm{H}_{29} \mathrm{~N}_{4} \mathrm{O}_{5}$ $[\mathrm{M}+\mathrm{H}]^{+}$: 489.2138 ; found: 489.2154 .

Methyl 4-acetyl-1-(4-chlorobenzyl)-1H-1,2,3-triazol-4-yl) methyl)-5-methyl-2-phenyl-1 $\mathrm{H}$-pyrrole-3-carboxylate (10a)

Yellow oil; yield 77\%; ${ }^{1} \mathrm{H}$ NMR $\left(400 \mathrm{MHz}, \mathrm{CDCl}_{3}\right)$ $\delta$ 7.42-7.33 (m, 5H), 7.24-7.21 (m, 2H), 7.00-6.96 (m, $2 \mathrm{H}), 6.92(\mathrm{~s}, 1 \mathrm{H}), 5.52(\mathrm{~s}, 2 \mathrm{H}), 4.98(\mathrm{~s}, 2 \mathrm{H}), 3.57(\mathrm{~s}, 3 \mathrm{H})$, $2.44(\mathrm{~s}, 3 \mathrm{H}), 2.41$ (s, 3H); ${ }^{13} \mathrm{C}$ NMR $\left(100 \mathrm{MHz}, \mathrm{CDCl}_{3}\right)$ $\delta$ 198.5, 165.4 162.5, 160.1, 143.8, 137.0, 133.4, 131.8, $131.7,130.7,130.0,129.0,128.3,123.4,122.0,113.3$, 51.4, 41.5, 39.8, 31.1, 11.5; HRMS (ESI-TOF) $\mathrm{m} / z$, calcd. for $\mathrm{C}_{25} \mathrm{H}_{24} \mathrm{ClN}_{4} \mathrm{O}_{3}[\mathrm{M}+\mathrm{H}]^{+}$: 463.1537 ; found: 463.1550 .

Methyl 4-acetyl-1-((1-(4-chlorobenzyl)-1H-1,2,3-triazol4-yl)methyl)-5-methyl-2-(4-methoxyphenyl)-1 H-pyrrole3-carboxylate (10b)

Yellow oil; yield 48\%; ${ }^{1} \mathrm{H}$ NMR $\left(400 \mathrm{MHz}, \mathrm{CDCl}_{3}\right.$ ) $\delta$ 7.37-7.35 (m, 4H), 7.15-7.10 (m, 4H), $6.86(\mathrm{~s}, 1 \mathrm{H}), 5.41$ (s, 2H), 4.99 (s, 2H), $3.82(\mathrm{~s}, 3 \mathrm{H}), 3.60$ (s, 3H), 2.42 (s, 3H), 2.40 (s, 3H); ${ }^{13} \mathrm{C}$ NMR (100 MHz, $\left.\mathrm{CDCl}_{3}\right) \delta 198.3$, 165.4, 160.0, 136.8, 134.2, 133.9, 133.0, 132.8, 131.9, $129.5,129.4,129.0,123.4,122.6,113.7,113.4,55.3,53.5$, 51.3, 39.8, 31.0, 11.5; HRMS (ESI-TOF) $\mathrm{m} / \mathrm{z}$, calcd. for $\mathrm{C}_{26} \mathrm{H}_{26} \mathrm{ClN}_{4} \mathrm{O}_{4}[\mathrm{M}+\mathrm{H}]^{+}:$493.1643; found: 493.1654 .

Methyl 4-acetyl-1-((1-octyl-1H-1,2,3-triazol-4-yl)methyl)5-methyl-2-phenyl-1H-pyrrole-3-carboxylate (11a)

Yellow oil; yield 62\%; ${ }^{1} \mathrm{H}$ NMR $\left(400 \mathrm{MHz}, \mathrm{CDCl}_{3}\right)$ $\delta$ 7.41-7.39 (m, 3H), 7.29-7.27 (m, 2H), $6.89(\mathrm{~s}, 1 \mathrm{H}), 5.02$ (s, 2H), $4.25(\mathrm{t}, 2 \mathrm{H}), 3.59(\mathrm{~s}, 3 \mathrm{H}), 2.43(\mathrm{~s}, 3 \mathrm{H}) 2.42(\mathrm{~s}, 3 \mathrm{H})$, $1.70(\mathrm{~s}, 2 \mathrm{H}), 1.27-1.25(\mathrm{~m}, 10 \mathrm{H}), 0.87(\mathrm{t}, 3 \mathrm{H}) ;{ }^{13} \mathrm{C}$ NMR $\left(100 \mathrm{MHz}, \mathrm{CDCl}_{3}\right) \delta 198.4,165.5,143.8,137.0,133.4$, $130.8,130.7,129.1,128.4,123.5,121.5,113.4,51.5,50.6$, 40.2, 31.8, 31.1, 30.3, 29.1, 29.0, 26.5, 22.7, 14.2, 11.6; HRMS (ESI-TOF) $\mathrm{m} / z$, calcd. for $\mathrm{C}_{26} \mathrm{H}_{35} \mathrm{~N}_{4} \mathrm{O}_{3}[\mathrm{M}+\mathrm{H}]^{+}$: 451.2709; found: 451.2726 .
Methyl 4-acetyl-1-((1-octyl-1H-1,2,3-triazol-4-yl)methyl)5-methyl-2-(4-methoxyphenyl)-1 $\mathrm{H}$-pyrrole-3-carboxylate (11b)

Yellow oil; yield $88 \%$; ${ }^{1} \mathrm{H}$ NMR (400 $\mathrm{MHz}, \mathrm{CDCl}_{3}$ ) $\delta$ 7.20-7.18 (m, 2H), 6.95 (s, 1H), 6.91-6.89 ( m, 2H), 5.0 $(\mathrm{s}, 2 \mathrm{H}), 4.24-4.23(\mathrm{~m}, 2 \mathrm{H}), 3.82-3.80(\mathrm{~m}, 3 \mathrm{H}), 3.60-3.58(\mathrm{~m}$, $3 \mathrm{H}), 2.40-2.39(\mathrm{~m}, 6 \mathrm{H}), 1.82-1.80(\mathrm{~m}, 2 \mathrm{H}), 1.24$ (bs, 10H), 0.87-0.84 (m, 3H); ${ }^{13} \mathrm{C}$ NMR (100 MHz, $\left.\mathrm{CDCl}_{3}\right) \delta 198.3$, 165.5, 160.1, 143.8, 137.0, 133.1, 132.0, 123.5, 122.7, 121.6, 113.8, 113.4, 55.3, 51.4, 50.6, 40.1, 31.7, 31.1, 30.3, 29.1, 29.0, 26.5, 22.6, 14.1, 11.5; HRMS (ESI-TOF) $\mathrm{m} / 2$, calcd. for $\mathrm{C}_{27} \mathrm{H}_{37} \mathrm{~N}_{4} \mathrm{O}_{4}[\mathrm{M}+\mathrm{H}]^{+}$: 481.2815; found: 481.2842.

Complete spectral data for compounds 8-11 are available in the SI section.

General procedure for the synthesis of 6-( $(1 H-1,2,3$-triazol4-yl)methyl)-2,6-dihydro-1 $H$-pyrrolo[3,4-d]pyridazin-1-ones (12-15)

Ethanol $(10 \mathrm{~mL})$, the respective methyl 4-acetyl1-((1H-1,2,3-triazol-4-yl)methyl)-1H-pyrrole3-carboxylate 8-11 (1 mmol), and hydrazine hydrochloride $(1.5 \mathrm{mmol}, 0.103 \mathrm{~g})$ were added to a round-bottomed flask at room temperature. The reaction mixture was magnetically stirred under reflux $\left(80^{\circ} \mathrm{C}\right)$ for $4 \mathrm{~h}$. Subsequently, ethanol and other volatile components were evaporated under reduced pressure, and the resulting solid was purified by recrystallization from ethanol (12-15).

6-((1-Benzyl-1H-1,2,3-triazol-4-yl)methyl)-4,5-dimethyl7-phenyl-2,6-dihydro-1 $H$-pyrrolo[3,4-d]pyridazin-1-one (12a)

White solid; yield 58\%; mp 175-181 ${ }^{\circ} \mathrm{C}$; ${ }^{1} \mathrm{H}$ NMR $\left(400 \mathrm{MHz}, \mathrm{CDCl}_{3}\right) \delta 8.95(\mathrm{~s}, 1 \mathrm{H}), 7.38-7.29(\mathrm{~m}, 8 \mathrm{H})$, 7.18-7.16 (m, 2H), $6.68(\mathrm{~s}, 1 \mathrm{H}), 5.41(\mathrm{~s}, 2 \mathrm{H}), 5.28(\mathrm{~s}, 2 \mathrm{H})$, $2.71(\mathrm{~s}, 3 \mathrm{H}), 2.51(\mathrm{~s}, 3 \mathrm{H}) ;{ }^{13} \mathrm{C}$ NMR $\left(100 \mathrm{MHz}, \mathrm{CDCl}_{3}\right)$ $\delta$ 159.0, 143.8, 142.2, 134.2, 132.2, 131.0, 130.1, 129.2, 129.0, 128.9, 128.3, 128.0, 125.4, 121.8, 116.6, 111.70, 54.3, 40.3, 21.0, 12.1; HRMS (ESI-TOF) $\mathrm{m} / \mathrm{z}$, calcd. for $\mathrm{C}_{24} \mathrm{H}_{23} \mathrm{~N}_{6} \mathrm{O}[\mathrm{M}+\mathrm{H}]^{+}:$411.1933; found: 411.1944 .

6-((1-Benzyl-1H-1,2,3-triazol-4-yl)methyl)-4,5-dimethyl7-(4-methoxyphenyl)-2,6-dihydro- $1 \mathrm{H}$-pyrrolo [3,4-d]pyridazin-1-one (12b)

White solid; yield 50\%; mp $178-181{ }^{\circ} \mathrm{C}$; ${ }^{1} \mathrm{H}$ NMR $\left(400 \mathrm{MHz}, \mathrm{CDCl}_{3}\right) \delta$ 7.39-7.37 (m, 2H), 7.20-7.18 (m, $2 \mathrm{H}), 7.10(\mathrm{~d}, 2 \mathrm{H}), 6.91-6.88(\mathrm{~m}, 1 \mathrm{H}), 6.83(\mathrm{~s}, 1 \mathrm{H}), 6.82(\mathrm{~d}$, 2H), 5.44 (s, 2H), 4.98 (s, 2H), 3.81 (s, 3H), 2.41 (s, 3H), 2.40 (s, 3H); ${ }^{13} \mathrm{C}$ NMR (100 MHz, $\left.\mathrm{CDCl}_{3}\right) \delta 160,144.3$, 134.4, 133.1, 132.0, 131.5, 129.3, 129.1, 128.7, 128.1, 127.0, 123.4, 122.7, 121.8, 114.0, 113.80, 55.4, 54.4, 40.0, 
27.9, 11.6; HRMS (ESI-TOF) $\mathrm{m} / 2$, calcd. for $\mathrm{C}_{25} \mathrm{H}_{25} \mathrm{~N}_{6} \mathrm{O}_{2}$ $[\mathrm{M}+\mathrm{H}]^{+}:$441.2039; found: 441.2050 .

6-((1-(4-Methoxybenzyl)-1H-1,2,3-triazol-4-yl) methyl)-4,5-dimethyl-7-phenyl-2,6-dihydro-1 $\mathrm{H}$-pyrrolo [3,4-d]pyridazin-1-one (13a)

White solid; yield 50\%; mp 153-159 ${ }^{\circ} \mathrm{C}$; ${ }^{1} \mathrm{H}$ NMR $\left(400 \mathrm{MHz}, \mathrm{CDCl}_{3}\right) \delta$ 7.35-7.29 (m, 5H), $7.14(\mathrm{~d}, 2 \mathrm{H})$, $6.89(\mathrm{~d}, 2 \mathrm{H}), 6.72(\mathrm{~s}, 1 \mathrm{H}), 5.34(\mathrm{~s}, 2 \mathrm{H}), 5.26(\mathrm{~s}, 2 \mathrm{H}), 3.81$ (s, 3H), $2.70(\mathrm{~s}, 3 \mathrm{H}), 2.52(\mathrm{~s}, 3 \mathrm{H}) ;{ }^{13} \mathrm{C}$ NMR $(100 \mathrm{MHz}$, $\left.\mathrm{CDCl}_{3}\right) \delta 160.0,159.0,143.8,142.3,132.2,131.0,130.1$, 129.8, 128.9, 128.4, 126.12, 125.5, 121.6, 116.7, 114.6, 111.70, 55.5, 53.9, 40.35, 21.17, 12.26; HRMS (ESI-TOF) $m / z$, calcd. for $\mathrm{C}_{25} \mathrm{H}_{25} \mathrm{~N}_{6} \mathrm{O}_{2}[\mathrm{M}+\mathrm{H}]^{+}: 441.2039$; found: 441.2042 .

6-((1-(4-Methoxybenzyl)-1H-1,2,3-triazol-4-yl)methyl)4,5-dimethyl-7-(4-methoxyphenyl)-2,6-dihydro$1 \mathrm{H}$-pyrrolo[3,4-d]pyridazin-1-one (13b)

White solid; yield $66 \%$; mp $145-153{ }^{\circ} \mathrm{C}$; ${ }^{1} \mathrm{H}$ NMR $\left(400 \mathrm{MHz}, \mathrm{CDCl}_{3}\right) \delta$ 7.38-7.27 (m, 4H), 7.16-7.12 (m, 2H), 6.90-6.86 (m, 2H), 6.73 (s, 1H), 5.41 (s, 2H), 4.99 (s, 2H), $3.82(\mathrm{~s}, 3 \mathrm{H}), 3.60(\mathrm{~s}, 3 \mathrm{H}), 2.42(\mathrm{~s}, 3 \mathrm{H}), 2.40(\mathrm{~s}$, $3 \mathrm{H}) ;{ }^{13} \mathrm{C}$ NMR $\left(100 \mathrm{MHz}, \mathrm{CDCl}_{3}\right) \delta 160,144.3,134.4$, 133.1, 132.0, 131.5, 129.3, 129.1, 128.7, 128.1, 127.0, 123.4, 122.7, 121.8, 114.0, 113.80, 55.5, 55.4, 53.9, 40.35, 21.17, 12.26; HRMS (ESI-TOF) $m / z$, calcd. for $\mathrm{C}_{26} \mathrm{H}_{27} \mathrm{~N}_{6} \mathrm{O}_{3}$ $[\mathrm{M}+\mathrm{H}]^{+}:$: 471.2145; found: 471.2157 .

6-((1-(4-Chlorobenzyl)-1H-1,2,3-triazol-4-yl)methyl)4,5-dimethyl-7-phenyl-2,6-dihydro- $1 \mathrm{H}$-pyrrolo [3,4-d]pyridazin-1-one (14a)

Yellow oil; yield 42\%; ${ }^{1} \mathrm{H}$ NMR (400 MHz, $\mathrm{CDCl}_{3}$ ) $\delta$ 7.35-7.32 (m, 7H), $7.14(\mathrm{~d}, 2 \mathrm{H}), 6.79(\mathrm{~s}, 1 \mathrm{H}), 5.40(\mathrm{~s}, 2 \mathrm{H})$, $5.30(\mathrm{~s}, 2 \mathrm{H}), 2.73$ (s, 3H), $2.54(\mathrm{~s}, 3 \mathrm{H}) ;{ }^{13} \mathrm{C} \mathrm{NMR}(100 \mathrm{MHz}$, $\left.\mathrm{CDCl}_{3}\right) \delta$ 159.0, 135.2, 134.0, 132.8, 131.4, 131.1, 129.8, $129.7,129.6,129.5,129.2,128.8,128.5,122.0,54.2,53.7$, 40.5, 29.8, 20.9, 12.4; HRMS (ESI-TOF) $\mathrm{m} / \mathrm{z}$, calcd. for $\mathrm{C}_{24} \mathrm{H}_{22} \mathrm{ClN}_{6} \mathrm{O}[\mathrm{M}+\mathrm{H}]^{+}:$:45.1544; found: 445.1556 .

6-((1-(4-Chlorobenzyl)-1H-1,2,3-triazol-4-yl)methyl)4,5-dimethyl-7-(4-methoxyphenyl)-2,6-dihydro$1 \mathrm{H}$-pyrrolo[3,4- $d$ ]pyridazin-1-one (14b)

Yellow oil; yield 46\%; ${ }^{1} \mathrm{H}$ NMR $\left(400 \mathrm{MHz}, \mathrm{CDCl}_{3}\right.$ ) $\delta$ 7.37-7.35 (m, 4H), $7.15(\mathrm{~d}, 2 \mathrm{H}), 7.11(\mathrm{~d}, 2 \mathrm{H}), 6.86(\mathrm{~s}$, $1 \mathrm{H}), 5.41(\mathrm{~s}, 2 \mathrm{H}), 4.99(\mathrm{~s}, 2 \mathrm{H}), 3.82(\mathrm{~s}, 3 \mathrm{H}), 2.42(\mathrm{~s}, 3 \mathrm{H})$, $2.40(\mathrm{~s}, 3 \mathrm{H}) ;{ }^{13} \mathrm{C}$ NMR $\left(100 \mathrm{MHz}, \mathrm{CDCl}_{3}\right) \delta 160.0,135.0$, $134.2,133.9,132.8,132.0,131.9,129.5,129.4,129.3$, 129.0, 122.6, 114.32, 113.7, 113.4, 55.3, 53.5, 39.8, 29.7, 11.5; HRMS (ESI-TOF) $\mathrm{m} / z$, calcd. for $\mathrm{C}_{25} \mathrm{H}_{24} \mathrm{ClN}_{6} \mathrm{O}_{2}$ $[\mathrm{M}+\mathrm{H}]^{+}:$475.1649; found: 475.1677 .
6-((1-Octyl-1H-1,2,3-triazol-4-yl)methyl)-4,5-dimethyl7-phenyl-2,6-dihydro-1 $H$-pyrrolo[3,4-d]pyridazin-1-one (15a)

Yellow oil; yield 40\%; ${ }^{1} \mathrm{H}$ NMR (400 $\mathrm{MHz}, \mathrm{CDCl}_{3}$ ) $\delta$ 7.44-7.42 (m, 5H), $6.83(\mathrm{~s}, 1 \mathrm{H}), 5.32(\mathrm{~s}, 2 \mathrm{H}), 4.25(\mathrm{t}, 2 \mathrm{H})$, $2.71(\mathrm{~s}, 3 \mathrm{H}), 2.53(\mathrm{~s}, 3 \mathrm{H}), 1.27-1.24(\mathrm{~m}, 12 \mathrm{H}), 0.87(\mathrm{t}, 3 \mathrm{H})$; ${ }^{13} \mathrm{C} \mathrm{NMR}\left(100 \mathrm{MHz}, \mathrm{CDCl}_{3}\right) \delta 159.1,143.6,142.4,132.5$, 131.5, 131.2, 129.1, 128.6, 128.6, 121.6, 116.9, 50.7, 40.6, 31.8, 30.3, 29.8, 29.2, 29.0, 26.5, 22.7, 21.1, 14.2, 12.3; HRMS (ESI-TOF) $m / z$, calcd. for $\mathrm{C}_{25} \mathrm{H}_{33} \mathrm{~N}_{6} \mathrm{O}[\mathrm{M}+\mathrm{H}]^{+}$: 433.2716; found: 433.2729 .

6-((1-Octyl-1H-1,2,3-triazol-4-yl)methyl)-4,5-dimethyl7-(4-methoxyphenyl)-2,6-dihydro-1 H-pyrrolo [3,4-d]pyridazin-1-one (15b)

Yellow oil; yield $70 \% ;{ }^{1} \mathrm{H}$ NMR $\left(400 \mathrm{MHz}, \mathrm{CDCl}_{3}\right) \delta$ $7.34(\mathrm{~d}, 2 \mathrm{H}), 7.01-6.97(\mathrm{~m}, 1 \mathrm{H}), 6.95(\mathrm{~d}, 2 \mathrm{H}), 5.30(\mathrm{~d}, 2 \mathrm{H})$, $4.26(\mathrm{t}, 2 \mathrm{H}), 3.82(\mathrm{~s}, 3 \mathrm{H}), 2.26(\mathrm{~s}, 3 \mathrm{H}), 2.51(\mathrm{~s}, 3 \mathrm{H}), 1.84-$ $1.81(\mathrm{~m}, 10 \mathrm{H}), 0.86(\mathrm{t}, 3 \mathrm{H}) ;{ }^{13} \mathrm{C} \mathrm{NMR}\left(100 \mathrm{MHz}, \mathrm{CDCl}_{3}\right)$ $\delta 160.1,159.3,152.4,143.7,141.8,132.8,132.8,132.4$, 122.2, 121.7, 116.6, 114.0, 111.4, 55.4, 50.7, 40.7, 31.8, $31.8,30.3,29.2,29.0,26.5,22.7,21.0,14.2,12.3$; HRMS (ESI-TOF) $m / z$, calcd. for $\mathrm{C}_{26} \mathrm{H}_{35} \mathrm{~N}_{6} \mathrm{O}_{2}[\mathrm{M}+\mathrm{H}]^{+}: 463.2821$; found: 463.2837 .

\section{Results and Discussion}

We started our research by using a convenient methodology for the synthesis of tetrasubstituted $1 H$-pyrroles 2a-c, which are the starting material for our proposed synthesis. Although many methodologies have been developed for the synthesis of pyrroles, it is still difficult to prepare tetrasubstituted $1 H$-pyrroles from easily accessible reagents. ${ }^{41}$ Thus, vinyl azides appear to be versatile precursors, as well as an alternative to classical methodologies, ${ }^{42-44}$ for obtaining the pyrroles considered herein. Firstly, following a well-known procedure, ${ }^{45}$ the three selected vinyl azides 1a-c were prepared from the reaction of methyl 2-azidoacetate with three arylaldehydes, and this resulted in substitution patterns in the phenyl rings due to the introduction of activating and deactivating groups to modulate opposite electronic effects. Subsequently, the pyrroles 2a-c (Scheme 2) could be obtained by refluxing a mixture of vinyl azides 1a-c and acetylacetone in toluene at $100{ }^{\circ} \mathrm{C}$ for $4 \mathrm{~h} .{ }^{45}$ Similar to the literature, the pyrroles 2a and 2b were obtained at a yield of 87 and $72 \%$, respectively, and by using the same methodology, the unpublished 2-(4-nitrophenyl)pyrrole $\mathbf{2 c}$ was synthesized at $63 \%$ yield. It is important to mention that the vinyl azide 1c decomposed in about a week, even when stored in a refrigerator. 


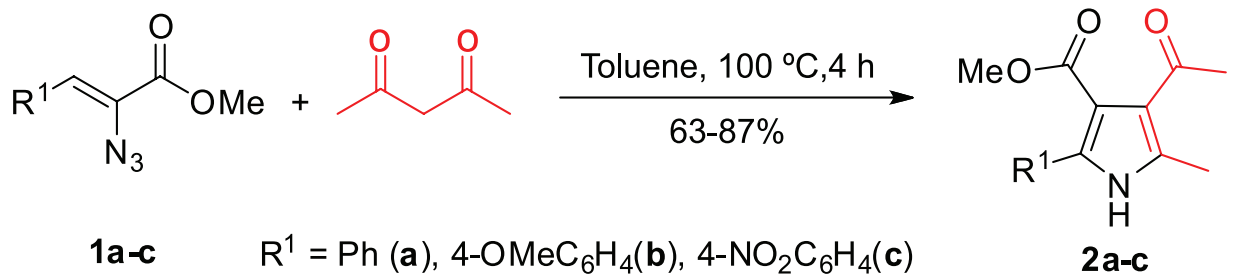

Scheme 2. Synthetic route to the tetrasubstituted $1 H$-pyrroles 2 a-c.

With the starting materials $\mathbf{2 a - c}$ in hand, compound 2a was selected as the standard pyrrole for optimization of the step-by-step reaction conditions. We initially turned our attention towards targeting an expedient protocol for the $\mathrm{N}$-alkylation reaction of $\mathbf{2 a}$ with propargyl bromide, in order to obtain the N-propargylated pyrrole $\mathbf{3 a}$ (see Scheme 3). A survey in the literature showed that a base would be necessary to promote the $\mathrm{N}$-deprotonation reaction of the $1 H$-pyrrole and solvents, and that temperature and reaction time must be optimized. It was found that $\mathrm{KOH}$ in acetone ${ }^{46}$ or $\mathrm{K}_{2} \mathrm{CO}_{3}$ in $\mathrm{DMF}^{47-49}$ could be used to achieve the desired product 3a.

When the N-propargylation reaction was conducted for $2 \mathbf{a}(1.0 \mathrm{mmol})$, using propargyl bromide $(1.2 \mathrm{mmol})$ in $\mathrm{KOH} /$ acetone medium at room temperature, product $\mathbf{3 a}$ was isolated at only $10 \%$ yield (Table 1 , entry 1 ). However, reactions conducted in DMF were the most suitable choice when combined with $\mathrm{K}_{2} \mathrm{CO}_{3}$ as base. The base $\mathrm{K}_{2} \mathrm{CO}_{3}$ was evaluated in 2.0, 4.0 and 6.0 equiv. It was found that the ratio of 4.0 equiv. of $\mathrm{K}_{2} \mathrm{CO}_{3}$ provided the best reaction condition. Thus, the reaction in DMF as solvent with either 4.0 or 6.0 equiv. of $\mathrm{K}_{2} \mathrm{CO}_{3}$, at room temperature for $14 \mathrm{~h}$, furnished the product $\mathbf{3 a}$ at the same yield of $87 \%$ (Table 1, entries 2 and 4). The conversion to $\mathbf{3 a}$ was followed by thin-layer chromatography (TLC) until complete consumption of the starting material 2a. However, when the same reaction conditions were tested at $65^{\circ} \mathrm{C},{ }^{48}$ the yield remained similar ( $85 \%$ ) to that obtained at room temperature, but the reaction time could be decreased to $5 \mathrm{~h}$ (Table 1, entry 5). Moreover, the reaction at $80^{\circ} \mathrm{C}$ gave a very similar yield (86\%)-see Table 1, entry 6 .

Thus, considering the good thermal stability of $\mathbf{3 a}$ at $65^{\circ} \mathrm{C}$, we chose the $\mathrm{N}$-alkylation reaction condition using DMF as solvent and 4.0 equiv. of $\mathrm{K}_{2} \mathrm{CO}_{3}$, at $65^{\circ} \mathrm{C}$ for $5 \mathrm{~h}$. Under this optimized condition, the new pyrroles $\mathbf{3 a}-\mathbf{c}$ were obtained at a yield of $85-87 \%$ (Scheme 3).

After establishing the best reaction condition for the first reaction step, we next turned our attention to the CuAAC reaction. From the independent discovery of Sharpless and co-workers ${ }^{24}$ in 2002 regarding the ability of $\mathrm{CuI}$ to catalyze the 1,3-dipolar cycloaddition reaction between a terminal alkyne and an organic azide, with regiospecific formation of 1,4-disubstituted 1,2,3-triazoles, the number of publications involving the synthesis of 1,2,3-triazoles has grown exponentially in several areas of chemistry. ${ }^{24,25}$ Many of these successful and versatile cycloaddition reactions have been catalyzed by $\mathrm{CuI}$ using 5 to $20 \mathrm{mmol} \%$, at reaction temperatures of $20-25{ }^{\circ} \mathrm{C}$ to

Table 1. Optimization of reaction conditions ${ }^{\text {a }}$ for the $N$-alkylation of pyrrole $\mathbf{2 a}$

\begin{tabular}{llllll} 
Solvent, base, \\
entry \\
\hline 1
\end{tabular}

Reaction conditions: pyrrole 2a $(1.0 \mathrm{mmol})$ and propargyl bromide $(1.2 \mathrm{mmol})$; bisolated yields; rt: room temperature; DMF: dimethylformamide. 


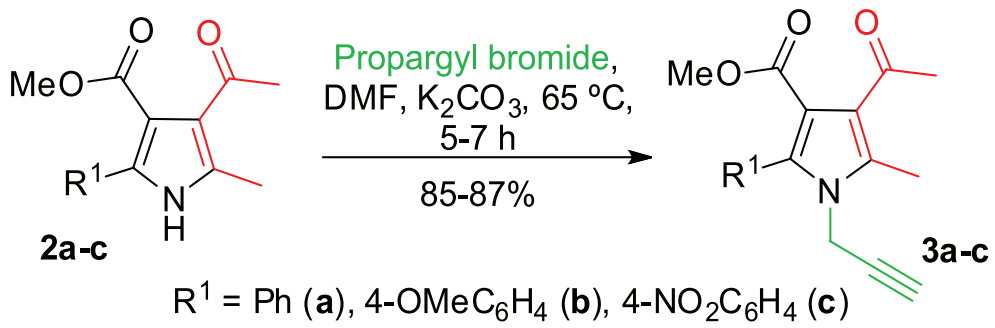

Scheme 3. Synthetic route to 1-(prop-2-yn-1-yl)-1H-pyrroles 3a-c.

around $120^{\circ} \mathrm{C} . .^{50-54}$ Thus, in order to attain triazole synthesis, similar processes in the literature related to our objective were investigated. Many of them use $t-\mathrm{BuOH} / \mathrm{H}_{2} \mathrm{O}$ mixtures as the solvent for this type of reaction, ${ }^{50-54}$ whereas others use the in situ formation of the organic azide to substitute the use of pre-formed organic azide, in order to avoid an additional reaction step. ${ }^{55,56}$ Thus, sodium azide and benzyl chloride were selected to be used for the in situ formation of the benzyl azide, and equimolar quantities of both compounds to the reactions were added. The reactions were calculated so that the amount of benzyl azide formed gave a 1:1.2 molar ratio between the pyrrole 3 and the benzyl azide.

In order to optimize the derivatization of pyrroles $\mathbf{3}$ for the triazolylmethyleno pyrrole system 8-11 via $\mathrm{CuAAC}$ reactions, pyrrole $\mathbf{3 a}$ was selected together with the standard reaction conditions described in the literature. ${ }^{52}$ The conversion of $\mathbf{3 a}$ to $\mathbf{8} \mathbf{a}$ was followed by TLC, until complete consumption of the starting material 3a-see Table 2 .

Table 2 shows that for reactions carried out using $5 \mathrm{mmol} \% \mathrm{CuI}$ at either $20-25$ or $80{ }^{\circ} \mathrm{C}$, and with reaction times of 5-12 h, the reaction yield did not exceed $61 \%$ (Table 1, entries 1-3). The best yields were obtained when the amount of catalyst (CuI) was increased to 10 and $15 \mathrm{mmol} \%$ in reactions conducted at temperatures of 80-95 ${ }^{\circ} \mathrm{C}$, which enabled reduction of the reaction time from $12 \mathrm{~h}$ to either 5 or $4 \mathrm{~h}$ (Table 2, entries 4-6).

Thus, when the reactions were performed at $80^{\circ} \mathrm{C}$ in a mixture of tert-butanol and water $(1: 1 \mathrm{v} / \mathrm{v})$ as solvent, with $15 \mathrm{mmol} \%$ of $\mathrm{CuI}$ as catalyst, $1.0 \mathrm{mmol}$ of $\mathbf{3 a}, 1.2 \mathrm{mmol}$ of sodium azide, and $1.2 \mathrm{mmol}$ of benzyl chloride, 8a was obtained at a yield of $77 \%$ after $4 \mathrm{~h}$ of reaction; however, depending on the substituents, reactions times of up to $5 \mathrm{~h}$ were necessary.

After establishing the best reaction condition for the second reaction step (CuAAC), the scope of the pyrroles (3a-c) and organic azides (4-7) was expanded, varying the $\mathrm{R}^{1}$-position with phenyl (3a) and 4-methoxyphenyl (3b), and the $\mathrm{R}^{2}$-position with a phenyl (4), 4-methoxyphenyl (5), 4-chlorophenyl (6), and $n-\mathrm{C}_{8} \mathrm{H}_{17}$ group (7), in order to analyze the behavior of these electron-donating or electronwithdrawing and a long alkyl side chain substrates in this new protocol. Unfortunately, pyrrole 3c was excluded due to the chemical instability of the vinyl azide 1c. As a result, the desired 4-acetyl-1-((1-aryl-1H-1,2,3-triazol-4-yl) methyl)-5-methyl-2-aryl-1H-pyrrole-3-carboxylates 8a-b,

Table 2. Optimization of reaction conditions $\mathrm{s}^{\mathrm{a}}$ for obtaining pyrrole $\mathbf{8 a}$
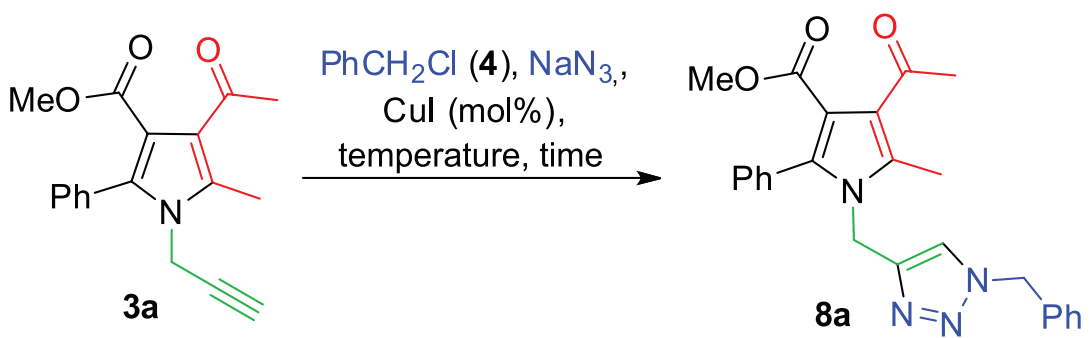

\begin{tabular}{|c|c|c|c|c|c|}
\hline entry & $\mathrm{NaN}_{3} / \mathrm{mmol}$ & $\mathrm{CuI} / \mathrm{mmol} \%$ & Temperature $/{ }^{\circ} \mathrm{C}$ & time $/ \mathrm{h}$ & Yield $^{\mathrm{b}} / \%$ \\
\hline 1 & 1.2 & 5 & $\mathrm{rt}$ & 12 & 60 \\
\hline 2 & 2.0 & 5 & $\mathrm{rt}$ & 12 & 61 \\
\hline 3 & 1.2 & 5 & 80 & 5 & 60 \\
\hline 4 & 1.2 & 10 & 80 & 5 & 65 \\
\hline 5 & 1.2 & 15 & 80 & 4 & 77 \\
\hline 6 & 1.2 & 15 & 95 & 4 & 78 \\
\hline
\end{tabular}

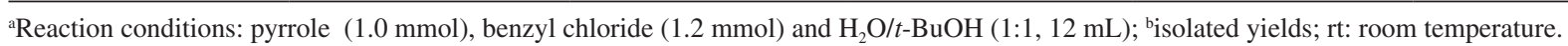


9a-b, 10a-b and 11a-b were obtained at moderate to good yields of $48-88 \%$ (see Scheme 4).

For the subsequent and last reaction step, the methyl 4-acetyl-1-((1-aryl(alkyl)-1H-1,2,3-triazol-4-yl)methyl)5-methyl-2-aryl-1H-pyrrole-3-carboxylates (8-11) were reacted with hydrazine monohydrochloride, in order to obtain the pyrrolo[3,4- $d]$ pyridazin-1-one system (12-15). Many protocols for the synthesis of $[3,4-d]$ pyridazin1-ones have used hydrazine hydrate or its hydrochloride salt in refluxing methanol or ethanol as the standard

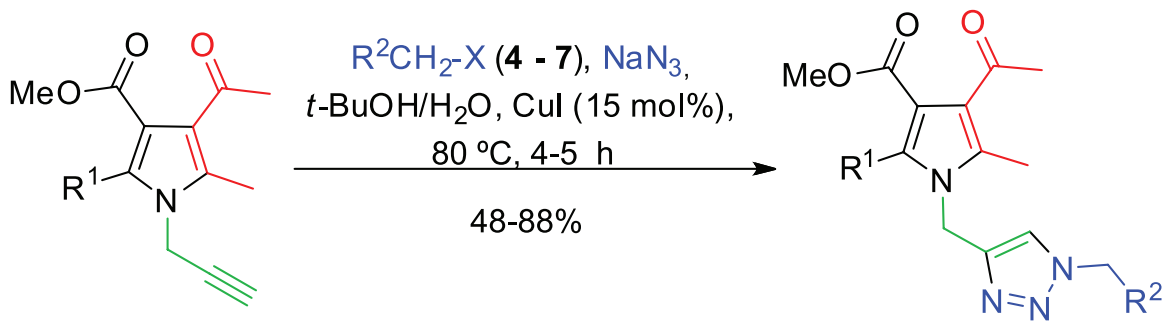

$3 a-b$

$8 a-b, 9 a-b, 10 a-b, 11 a-b$

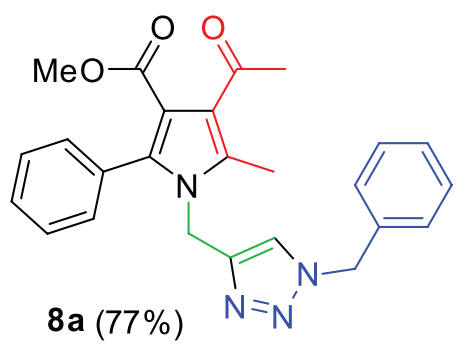<smiles>COC(=O)c1c(C(C)=O)c(C)n(Cc2cn(Cc3ccccc3)nn2)c1-c1ccc(OC(C)(C)C)cc1</smiles><smiles>COC(=O)c1c(C(C)=O)c(C)n(Cc2cn(Cc3ccc(OC)cc3)nn2)c1-c1ccccc1</smiles><smiles>COC(=O)c1c(C(C)=O)c(C)n(Cc2cn(Cc3ccc(OC)cc3)nn2)c1-c1ccc(OC)cc1</smiles><smiles>COC(=O)c1c(C(C)=O)c(C)n(Cc2cn(Cc3ccc(Cl)cc3)nn2)c1-c1ccccc1</smiles><smiles>COC(=O)c1c(C(C)=O)c(C)n(Cc2cn(Cc3ccc(Cl)cc3)nn2)c1-c1ccc(OC)cc1</smiles>

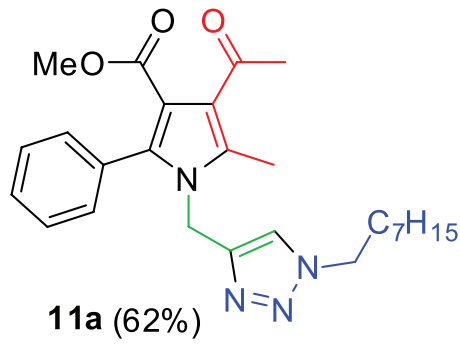<smiles>COC(=O)c1c(C(C)=O)c(C)n(Cc2cn(CC(C)C)nn2)c1-c1ccc(OC)cc1</smiles>

Scheme 4. Novel methyl 4-acetyl-1-((1H-1,2,3-triazol-4-yl)methyl)-1H-pyrrole 3-carboxylates (8a-b-11a-b). 
condition. ${ }^{57-59}$ Thus, the tests were performed using one of these solvents. The reaction of $\mathbf{8} \mathbf{a}$ and hydrazine hydrochloride in the appropriate solvent under reflux was followed by TLC until conversion to pyrrole $\mathbf{8 a}$ was verified. After some initial tests, we found that when the reaction of $\mathbf{8 a}$ with hydrazine hydrochloride at a 1:1.5 molar ratio was carried out in ethanol at reflux for $4 \mathrm{~h}$, it furnished the pyrrolo[3,4- $d$ ] pyridazin-1-one 12a at a satisfactory yield (58\%).

Knowing the optimized conditions for the third reaction step $([4+2]$ cyclocondensation reaction), and in order to analyze the behavior of the electron-donating or electronwithdrawing and the long $n$-alkylated chain substrates in this method, the scope for the final pyrrole system (12-15) was also expanded. As a result, the desired 6-((1H-1,2,3-triazol4-yl)methyl)-2,6-dihydro-1 $H$-pyrrolo[3,4- $d$ ]pyridazin1-ones 12a-b, 13a-b, 14a-b and 15a-b were also obtained at moderate to good yields of 40-70\% (Scheme 5). Again, it was observed that the reaction time to convert compounds 8-11 into 12-15 was dependent on the substituents attached to both rings, which resulted in reaction times of 4 to $5 \mathrm{~h}$. In summary, the yields of the reactions can not be predict because different products can be obtained from the methodology developed in this work due to structural variation of the starting materials.

Complementary, to demonstrate that the pyrrolo [3,4- $d$ ]pyridazin-1-ones $\mathbf{1 2 - 1 5}$ could be obtained regiospecifically and directly from the reaction of pyrroles $\mathbf{2}$, the synthesis of the selected pyridazinone 12a was also attempted by a one-pot three-steps reaction without the isolation of the propargylated pyrrole $\mathbf{3 a}$ and the triazole derivative $\mathbf{8 a}$, starting from pure $\mathbf{2 a}$ (Scheme 6).

Unfortunately and after several attempts, it became clear for us that only the first two reaction steps are feasible in DMF as optimized solvent, which led to the isolation of triazole 8a in only $40 \%$ yield from $\mathbf{2 a}$, without the isolation of 3a. Sequential one-pot reactions to obtain 12a directly from $2 \mathbf{a}$ or from $\mathbf{3 a}$, without the isolation of $\mathbf{8 a}$, resulted in mixtures of by-products of impossible separation through column chromatography.

All the new products $\mathbf{2 c}$, 3a-c, and 8-15 were fully characterized based on GC-MS and ${ }^{1} \mathrm{H} /{ }^{13} \mathrm{C}$ NMR spectroscopic data, as well as elemental analysis or HRMS data.

The structures of $\mathbf{3 a}$ and $\mathbf{8 a}$ were also unequivocally confirmed by single crystal X-ray diffraction, as shown in Figures 2 and 3, respectively. ${ }^{37,40}$ Crystal data and structure refinement parameters for molecules $\mathbf{3 a}$ and $\mathbf{8 a}$ are listed on Tables S1 and S4 (SI section), selected bond distances and angles observed are listed on Tables S2 and S5 (SI section) and interplanar angles between selected molecular fragments are listed on Tables S3 and S6, respectively (SI section).

Crystals of compound 3a and $\mathbf{8 a}$ are monoclinic and triclinic with respective space groups $C c$ and $P(-1)$. The structure analysis reveals both molecules with the respective site symmetry 1 . In the particular case of compound the acentric space group $C c$ was unequivocally determined and the attempt to solve the structure with the centrosymmetric space group $C 2 / c$ was not successful. Since the absolute structure parameter deviates from zero, racemic twinning in the crystal structure of is present. Due to the fact that the correct configuration of the molecular structure is not certain, the authors decided by the structure refinement considering the crystal to be twinned, using the TWIN command and thus eliminating the value corresponding to the absolute structure parameter while the batch scale factor parameter converges to the 0.19207 value (Table S1, SI section).

Geometrically, molecules 3a and 8a shows that the interplanar angles between that planes corresponding to the substituents, respectively, attached to the $\mathrm{N}(1)$, $C(2), C(3)$ and $C(4)$ atoms with the plane of the central pyrrole ring deviate significantly from the co-planarity (Tables S3 and S6, SI section). In this context, it is noteworthy in the molecule that the carbonyl group of the acetyl substituent connected to the $\mathrm{C}(4)$ atom of the central pyrrole ring is oriented in the same direction of the methyl substituent attached to the $\mathrm{C}(5)$ atom whereas in the molecule the carbonyl group of the acetyl substituent is oriented in opposite direction of the methyl substituent. This observation can be explained by the fact that in molecule 8a, the oxygen atom of the acetyl substituent involves an interaction with a carbon atom of the phenyl substituent of the $\pi-\pi \mathrm{O} \cdots \mathrm{C}$ type $\left[\mathrm{C}(25) \cdot \mathrm{O}(411)^{\# 3}\right.$ distance $=3.382(3) \AA$; symmetry code $(\# 3): 1+x, y, z]$. This interaction contributes to the formation of a onedimensional chain by translation of the molecule along the [100] crystallographic direction in the unit cell in $C c$ of compound in the solid state, as well as explains the non-coplanarity of the phenyl substituent with the central pyrrole ring (interplanar angle of $44.61(7)^{\circ}$, Table S3 (SI section) and Figure 4).

On the other hand, after the "click-reaction" on the ethynyl substituent of with phenyl-methylene-azide giving raise to the molecule, a terminal phenyl substituent results in the structure [( $\mathrm{C}(152)-\mathrm{C}(157)$-ring]. This phenyl ring is responsible for the observation of $\pi-\pi \mathrm{C} \cdots \mathrm{C}$ interactions including carbon atoms belonging to the central pyrrole ring and the phenyl substituent attached to its $\mathrm{C}(2)$ atom $\left[\mathrm{C}(2) \cdots \mathrm{C}(155)^{\# 7}\right.$ distance $=3.411(2) \AA$; $\mathrm{C}(3) \cdots \mathrm{C}(155)^{\# 7}$ distance $=3.475(2) \AA ; \mathrm{C}(22) \cdots \mathrm{C}(156)^{\# 7}$ 


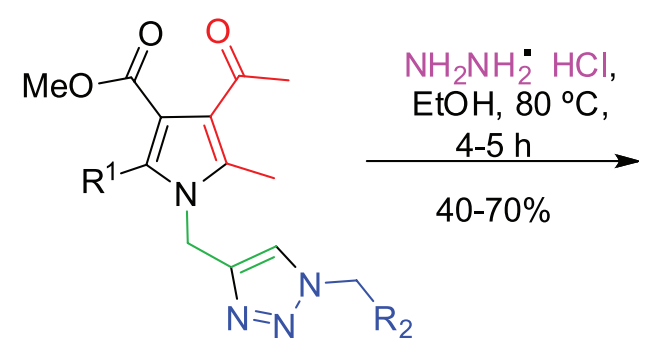

$8 a-b, 9 a-b, 10 a-b, 11 a-b$<smiles>[R]Cn1cc(Cn2c(C)c3c(C)n[nH]c(=O)c3c2[R])nn1</smiles>

12a-b, 13a-b, 14a-b, 15a-b<smiles>COc1ccc(-c2c3c(=O)[nH]nc(C)c3c(C)n2Cc2cn(Cc3ccccc3)nn2)cc1</smiles><smiles>COc1ccc(Cn2cc(Cn3c(C)c4c(C)n[nH]c(=O)c4c3-c3ccccc3)nn2)cc1</smiles><smiles>COc1ccc(Cn2cc(Cn3c(C)c4c(C)n[nH]c(=O)c4c3-c3ccc(OC)cc3)nn2)cc1</smiles><smiles>Cc1n[nH]c(=O)c2c(-c3ccccc3)n(Cc3cn(Cc4ccc(Cl)cc4)nn3)c(C)c12</smiles><smiles>COc1ccc(-c2c3c(=O)[nH]nc(C)c3c(C)n2Cc2cn(Cc3ccc(Cl)cc3)nn2)cc1</smiles><smiles>Cc1n[nH]c(=O)c2c(-c3ccccc3)n(Cc3cn(CCCCCCCCCC(C)(C)C)nn3)c(C)c12</smiles>

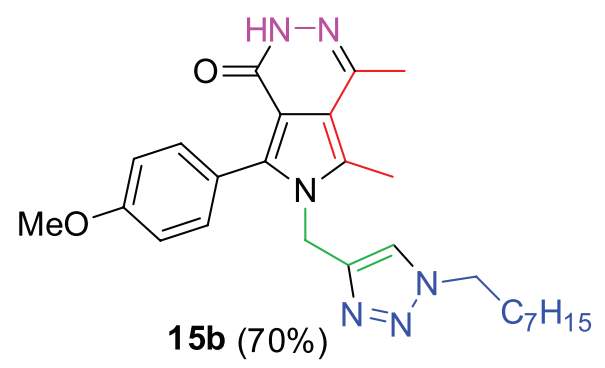

Scheme 5. Novel 6-((1H-1,2,3-triazol-4-yl)methyl)-2,6-dihydro-1H-pyrrolo[3,4- $d$ ]pyridazin-1-ones (12-15).

distance $=3.493(2) \AA$; symmetry code (\#7): $1+x, 1+y, z]$. As a consequence of this preferential interaction, the $\mathrm{O}(412)$ atom of the acetyl group was excluded from an interaction with the phenyl ring attached to $\mathrm{C}(2)$ atom of the central pyrrole ring, allowing its rearrangement by turning around the $\mathrm{C}(4)-\mathrm{C}(41)$ bond in the same direction 
<smiles>COC(=O)c1c(-c2ccccc2)[nH]c(C)c1C(C)=O</smiles>

2a

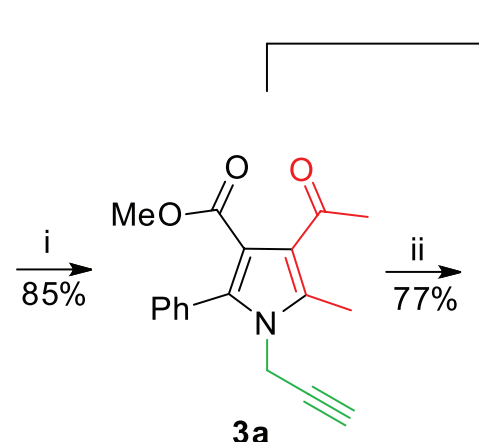

$3 a$ ii, iii $X$

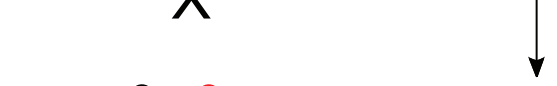

$8 a$<smiles>COC(=O)c1c(C(C)=O)c(C)n(Cc2cn(Cc3ccccc3)nn2)c1-c1ccccc1</smiles><smiles>Cc1n[nH]c(=O)c2c(-c3ccccc3)n(Cc3cn(Cc4ccccc4)nn3)c(C)c12</smiles>

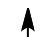

i, ii, iii

Reagents and Conditions: (i) = Propargyl bromide, $\mathrm{DMF}, \mathrm{K}_{2} \mathrm{CO}_{3}, 65^{\circ} \mathrm{C}, 5 \mathrm{~h}$; (ii) $=\mathrm{PhCH}_{2} \mathrm{Cl}, \mathrm{NaN}_{3}$, DMF, Cul $(15 \mathrm{~mol} \%), 80^{\circ} \mathrm{C}, 4 \mathrm{~h}$; (iii) $=\mathrm{NH}_{2} \mathrm{NH}_{2} \cdot \mathrm{HCl}$, DMF, $80^{\circ} \mathrm{C}, 5 \mathrm{~h}$.

Scheme 6. Sequential three-steps one-pot reaction to obtain 6-((1H-1,2,3-triazol-4-yl)methyl)-2,6-dihydro-1H-pyrrolo[3,4- $d]$ pyridazin-1-one 12a.

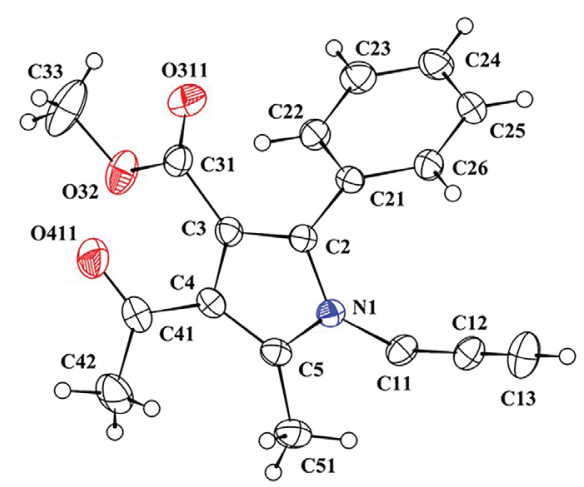

Figure 2. Projection of the molecular structure with atom-labeling scheme of 1-(prop-2-yn-1-yl)-1H-pyrrole (3a) (CCDC 1056078, SI section). Displacement ellipsoids at the $30 \%$ level. $\mathrm{H}$ atoms involve arbitrary radii.

to the methyl substituent attached to the $\mathrm{C}(5)$ atom of the central ring of molecule (Figure 5).

\section{Conclusions}

In summary, we successfully developed a streamlined new three-step methodology for the synthesis of a series of eight examples of polysubstituted 6-((1H-1,2,3-triazol-

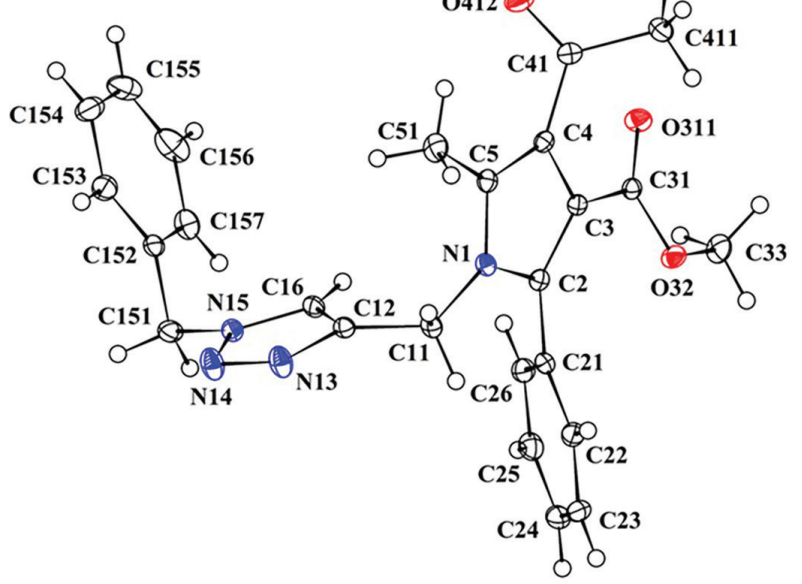

Figure 3. Projection of the molecular structure with atom-labeling scheme of 1-((1H-1,2,3-triazol-4-yl)methyl)-1H-pyrrole (8a) (CCDC 1574331, SI section).Displacement ellipsoids at the $50 \%$ level. $\mathrm{H}$ atoms involve arbitrary radii.

4-yl)methyl)-2,6-dihydro-1 $H$-pyrrolo[3,4- $d]$ pyridazin1 -ones, through the use of: sequential $N$-alkylation reactions of tetrasubstituted $\mathrm{NH}-$ pyrroles, which furnished a series of three examples of 1-(prop-2-yn-

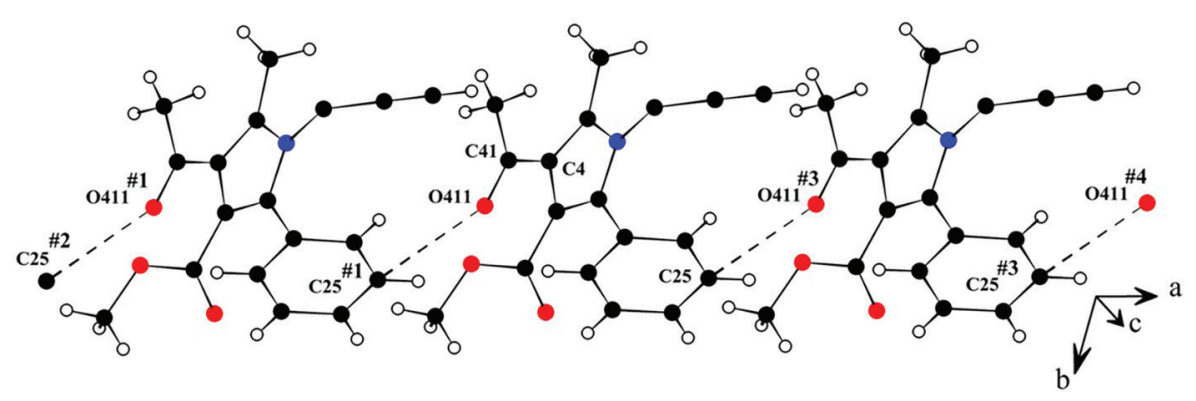

Figure 4. Section of the crystal structure packing of involving the one-dimensional arrangement by translation of molecules along the [100] crystallographic direction highlighting the $\pi-\pi \mathrm{O} \cdots \mathrm{C}$ interactions. Symmetry codes \#1: $-1+x, y, z ; \# 2:-2+x, y, z ; \# 3: 1+x, y, z ; \# 4: 2+x, y, z$. 


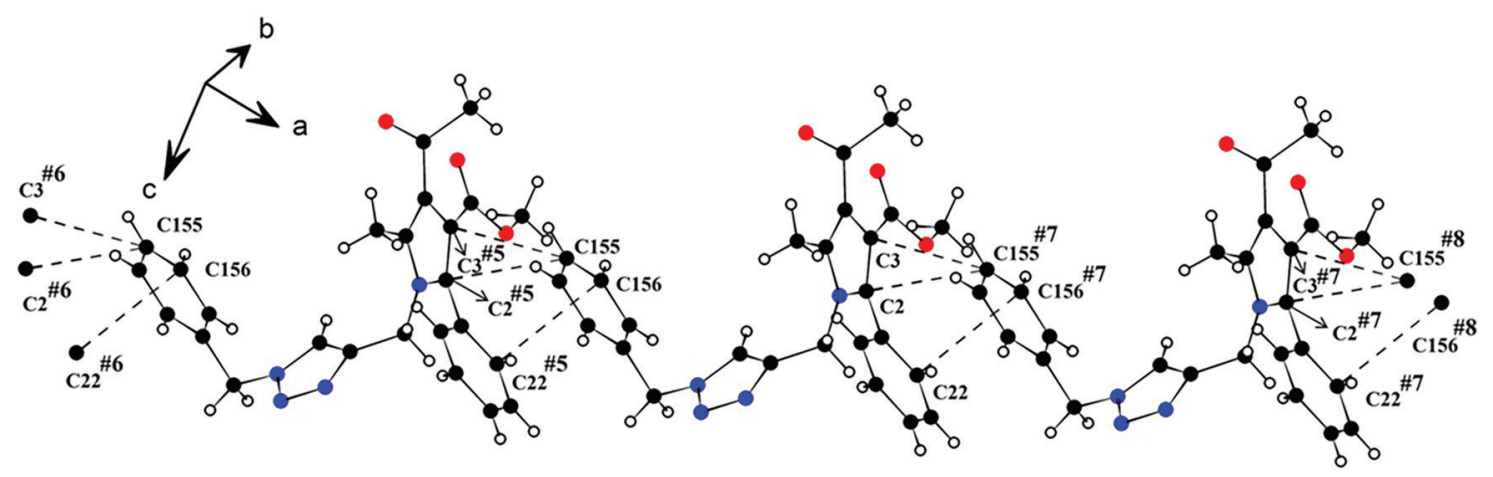

Figure 5. Section of the crystal structure packing of involving the one-dimensional arrangement by translation of molecules along the [110] crystallographic direction highlighting the $\pi-\pi \mathrm{C} \cdots \mathrm{C}$ interactions. Symmetry codes \#5: $-1+x,-1+y, z ; \# 6:-2+x,-2+y, z ; \# 7: 1+x, 1+y, z ; \# 8: 2+x, 2+y, z$.

1-yl)-1H-pyrroles; standard CuAAC “click chemistry", which involved some alkyl and aryl organic azides and $\mathrm{N}$-propargylated pyrroles to give eight examples of methyl 1-((1H-1,2,3-triazol-4-yl)methyl)-1H-pyrrole-3carboxylates; and [4 +2] cyclocondensation reactions of the respective ketoesters with hydrazine hydrochloride to furnish the pyrrolo[3,4- $d]$ pyridazin-1-one system under mild reaction conditions and at moderate to satisfactory yields. The aforementioned protocol could be applicable to a range of substrates and provide more complex and stable heterocyclic structures at yields up to $54 \%$, thus demonstrating the generality of this methodology. Other advantages of this synthetic route are smooth reaction conditions and readily available raw materials. The novel compounds obtained are currently being evaluated for their biological activity.

\section{Supplementary Information}

The crystallographic data for the structures of pyrroles 3a and 8a have been deposited with the Cambridge Crystallographic Data Centre and allocated the deposition numbers CCDC 1056078 and 1574331, respectively. Copies of the data can be obtained free of charge, upon application to: CCDC, 12 Union Road, Cambridge CB2 1EZ, UK. (fax: +441223336033 or deposit@ccdc.com.ac.uk).

Supplementary information (NMR spectral and $\mathrm{X}$-ray diffraction data) is available free of charge at http://jbcs.sbq.org.br as a PDF file.

\section{Acknowledgments}

The authors thank the Coordination for Improvement of Higher Education Personnel (CAPES) for the fellowships, as well as the National Council for Scientific and Technological Development (CNPq) for the financial support (process No. 306.883/2015-5 and 402.075/2016-1 $\mathrm{CNPq} /$ Universal).

\section{References}

1. Fox, B. M.; Iio, K.; Li, K.; Cho, R.; Takashi, I.; Jackson, S.; Sagawa, S.; Shan, B.; Tanaka, M.; Yoshida, A.; Kayser, F.; Bioorg. Med. Chem. Lett. 2010, 20, 6030.

2. Anker, N. B.; Arruda, J. M.; Campbell, B. T.; Munoz, B.; Petpiboon Prasit, Stearns, B. A.; Merck \& Co. Inc.; PTC Int. Appl. WO 2004006836 A2, 2003.

3. Bantick, J.; Cooper, M.; Thorne, P.; Perry, M.; PTC Int. Appl. WO 9929695 Al, 1999.

4. Cho, T. P.; Jun, F.; Li, H.; Zhe, X.; Ling, C.; Xu, Z.; Lei, Z.; Bing, H.; Bioorg. Med. Chem. Lett. 2009, 19, 6437.

5. Zwick, E.; Bange, J.; Ulrich, A.; Endocr-Relat. Cancer 2001, $8,161$.

6. Malinka, W.; Redzicka, A.; Wiesek, M. J.; Filipek, B.; Dybala, M.; Karczmarzyk, Z.; Lipkowska, Z. U.; Kalicki, P.; Eur. J. Med. Chem. 2011, 46, 4992.

7. Malinka, W.; Redzicka, A.; Lozach, O.; Il Farmaco 2004, 59, 457.

8. Malinka, W.; Pharmazie 2001, 56, 384.

9. Stearns, B. A.; Anker, N.; Arruda, J. M.; Campbell, B. T.; Chen, C.; Cramer, M.; Hu, T.; Jiang, X.; Park, K.; Ren, K. K.; Sablad, M.; Santini, A.; Schaffhauser, H.; Urban, M. O.; Munoz, B.; Bioorg. Med. Chem. Lett. 2004, 14, 1295.

10. Malinka, W.; Redzicka, A.; Jastrzebska-Wiesek, M.; Filipek, B.; Pol. 4pp. PL 212011, 2012.

11. Bantick, J.; Cooper, M.; Thorne, P.; Perry, M.; PCT Int. Appl. WO 9929695 Al, 1999.

12. Katritzky, A. R.; Rees, C. W.; Comprehensive Heterocyclic Chemistry: Structure, Reactions, Synthesis, and Uses of Heterocyclic Compounds; Pergamon Press: Oxford, 1984.

13. Kokilambigai, K. S.; Seetharaman, R.; Lakshmi, K. S.; Crit. Rev. Anal. Chem. 2017, 47, 538.

14. Hajkova, M.; Kratochvil, B.; Radl, S.; Chem. Listy 2008, 102, 3.

15. Kolb, H. C.; Sharpless, K. B.; Drug Discovery Today 2003, 8, 1128.

16. Xie, J.; Seto, C. T.; Bioorg. Med. Chem. 2007, 15, 458. 
17. Tornøe, C. W.; Christensen, C.; Meldal, M.; J. Org. Chem. 2002, 67, 3057.

18. Santos, J. O.; Pereira, G. R.; Brandão, G. C.; Borgati, T. F.; Arantes, L. M.; de Paula, R. C.; Soares, L. F.; do Nascimento, M. F. A.; Ferreira, M. R. C.; Taranto, A. G.; Varotti, F. P.; de Oliveira, A. B.; J. Braz. Chem. Soc. 2016, 27, 551.

19. Cassamale, T. B.; Costa, E. C.; Carvalho, D. B.; Cassemiro, N. S.; Tomazela, C. C.; Marques, M. C. S.; Ojeda, M.; Matos, M. F. C.; Albuquerque, S.; Arruda, C. C. P.; Baroni, A. C. M.; J. Braz. Chem. Soc. 2016, 27, 1217.

20. Silva Jr., E. N.; Jardim, G. A. M.; Menna-Barreto, R. F. S.; de Castro, S. L.; J. Braz. Chem. Soc. 2014, 25, 1780.

21. Aleixo, M. A. A.; Garcia, T. M.; Carvalho, D. B.; Viana, L. H.; Amaral, M. S.; Kassab, N. M.; Cunha, M. C.; Pereira, I. C.; Guerrero Jr., P. G.; Perdomo, R. T.; Matos, M. F. C.; Baroni, A. C. M.; J. Braz. Chem. Soc. 2018, 29, 109.

22. Torres, F. C.; Gonçalves, G. A.; Vanzolini, K. L.; Merlo, A. A.; Gauer, B.; Holzschuh, M.; Andrade, S.; Piedade, M.; Garcia, S. C.; Carvalho, I.; von Poser, G. L.; Kawano, D. F.; Eifler-Lima, V. L.; Cass, Q. B.; J. Braz. Chem. Soc. 2016, 27, 1541.

23. Dheer, D.; Singh, V.; Shankar, R.; Bioorg. Chem. 2017, 71, 30 and references therein.

24. Rostovtsev, V. V.; Green, L. G.; Fokin, V. V.; Sharpless, K. B.; Angew. Chem., Int. Ed. 2002, 41, 2596.

25. Worell, B. T. J.A.; Malik, J.A.; Fokin, V.V.; Science 2013, 340, 457.

26. Bonacorso, H. G.; Moraes, M. C.; Luz, F. M.; Quintana, P. S.; Zanatta, N.; Martins, M. A. P.; Tetrahedron Lett. 2015, 56, 441.

27. Bonacorso, H. G.; Ketzer, A.; Rosa, W. C.; Calheiro, T. P.; Rodrigues, M. B.; Zanatta, N.; Martins, M. A. P.; Frizzo, C. P.; J. Fluorine Chem. 2018, 210, 142.

28. Zanatta, N.; Schneider, J. M. F. M.; Schneider, P. H.; Wouters, A. D.; Bonacorso, H. G.; Martins, M. A. P.; Wessjohann, L. A.; J. Org. Chem. 2006, 71, 6996.

29. Zanatta, N.; Wouters, A. D.; Fantinel, L.; da Silva, F. M.; Barichello, R.; da Silva, P. E. A.; Ramos, D. F.; Bonacorso, H. G.; Martins, M. A. P.; Synlett 2009, 5, 755.

30. Zanatta, N.; Aquino, E. C.; da Silva, F. M.; Bonacorso, H. G.; Martins, M. A. P.; Synthesis 2012, 44, 3477.

31. Aquino, E. C.; Leonel, G.; Gariboti, V. C.; Frizzo, C. P.; Martins, M. A. P.; Bonacorso, H. G.; Martins, M. A. P.; J. Org. Chem. 2015, 80, 12453.

32. Bonacorso, H. G.; Libero, F. M.; dal Forno, G. M.; Pittaluga, E. P.; Back, D. F.; Hörner, M.; Martins, M. A. P.; Zanatta, N.; Tetrahedron Lett. 2016, 57, 4568.

33. Bonacorso, H. G.; Libero, F. M.; dal Forno, G. M.; Pittaluga, E. P.; Porte, L. M. F.; Martins, A. P.; Zanatta, N.; Tetrahedron Lett. 2015, 56, 5190.

34. DOC-M86-EXX229 APEX3 Software User Manual Bruker AXS Inc., Madison, Wisconsin, USA, 2016.

35. Bruker SAINT-V8.32A Data Reduction Software, Bruker AXS Inc., Madison, Wisconsin, USA, 2013.
36. Sheldrick, G. M.; SADABS: Program for Empirical Absorption Correction, University of Gottingen, Germany, 1996.

37. Burla, M. C.; Caliandro, R.; Carrozzini, B.; Cascarano, G. L.; Cuocci, C.; Giacovazzo, C.; Mallano, M.; Mazzone, A.; Polidori, G.; J. Appl. Crystallogr. 2015, 48, 306.

38. Sheldrick, G. M.; Acta Cryst. 2008, A64, 112.

39. Farrugia, L. J.; J. Appl. Cryst. 2012, 45, 849.

40. Brandenburg, K.; DIAMOND 3.1a., version 1.1a, Crystal Impact GbR, Bonn, Germany, 1997-2005.

41. Estéves, V.; Villacampa, M.; Menéndez, J. C.; Chem. Soc. Rev. 2014, 43, 4633.

42. Eicher, T.; Hauptmann, S.; The Chemistry of Heterocycles, $2^{\text {nd }}$ ed.; Wiley: London, 2003.

43. Knorr, L.; Ber. Dtsch. Chem. Ges. 1884, 17, 1635.

44. Paal, C.; Ber. Dtsch. Chem. Ges. 1885, 18, 367.

45. Chiba, S.; Feng Y.; Lapointe,W. G.; Narasaka, K.; Org. Lett. 2008, 10, 313 .

46. Prandi, C.; Occhiato, E. G.; Tabasso, S.; Bonfante, P.; Novero, M.; Scarpi, D.; Bova, M. E.; Miletto, I.; Eur. J. Org. Chem. 2011, 20, 3781.

47. Naveen, P.; Nikhila, G.; Udayakumar, D.; Venkat, K.; Perumal, Y.; Dharmarajan, S.; Med. Chem. Res. 2016, 25, 135.

48. Shyma, P. C.; Balakrishna, K.; Peethambar, S. K.; Vijesh, A. M.; Med. Chem. Res. 2016, 25, 2680.

49. Loaiza, P. R.; Löber, S.; Hübner, H.; Gmeiner, P.; Bioorg. Med. Chem. 2007, 15, 7248.

50. Aucagne, V.; Hänni, K. D.; Leigh, D. A.; Lusby, P. J.; Walker, D. B.; J. Am. Chem. Soc. 2006, 128, 2186.

51. Sreedhar, B.; Reddy, P. S.; Synth. Commun. 2007, 37, 805.

52. Kuang, G. C.; Michaels, H. A.; Simmons, J. T.; Clark, R. J.; Zhu, L.; J. Org. Chem. 2010, 75, 6540.

53. Trabocchi, A.; Menchi, G.; Cini, N.; Bianchini, F.; Raspanti, S.; Bottoncetti, A.; Pupi, A.; Calorini, L.; Guarna, A.; J. Med. Chem. 2010, 53, 7119.

54. Wu, P.; Feldman, A. K.; Nugent, A. K.; Hawker, C. J.; Scheel, A.; Voit, B.; Pyun, J.; Fréchet, J. M. J.; Sharpless, K. B.; Fokin V. V.; Angew. Chem., Int. Ed. 2004, 43, 3928.

55. Feldman, A. K.; Colasson, B.; Fokin, V. V.; Org. Lett. 2004, 22, 3897.

56. Scriven, E. F. V.; Turnbull, K.; Chem. Rev. 1988, 88, 297.

57. Gonçalves, D. S.; Silva, M. J. V.; Souza, T. F.; Jacomini, A. P.; Back, D. F.; Basso, E. A.; Moura, S.; Rosa, F. A.; Synthesis 2016, 48, 3042.

58. Naveen, P.; Nikhila, G.; Udayakumar, D.; Pulla, V. K.; Perumal, Y.; Dharmarajan, S.; Med. Chem. Res. 2016, 25, 135.

59. Shyma, P. C.; Balakrishna, K.; Peethambar, S. K.; Vijesh, A. M.; Med. Chem. Res. 2016, 25, 2680.

Submitted: September 18, 2018

Published online: January 29, 2019 